\title{
The UNIT algorithm for solving one-dimensional convection-diffusion problems via integral transforms ${ }^{\text {is }}$
}

\author{
L.A. Sphaier ${ }^{\text {a,* }}$, R.M. Cotta ${ }^{\text {b }}$, C.P. Naveira-Cotta ${ }^{\text {b }}$, J.N.N. Quaresma ${ }^{\text {c }}$ \\ a Laboratory of Theoretical and Applied Mechanics - LMTA, Department of Mechanical Engineering, Universidade Federal Fluminense - PGMEC/UFF, \\ Rua Passo da Pátria 156, bloco E, sala 216, Niterói, RJ, 24210-240, Brazil \\ ${ }^{\mathrm{b}}$ Laboratory of Transmission and Technology of Heat - LTTC, Department of Mechanical Engineering, Universidade Federal do Rio de Janeiro - COPPE/UFRJ, \\ Cx. Postal 68503, Cidade Universitária - Rio de Janeiro, RJ, 21945-970, Brazil \\ c School of Chemical Engineering, Universidade Federal do Pará - FEQ/UFPA, Campus Universitário do Guamá, 66075-110, Belém, PA, Brazil
}

\section{A R T I C L E I N F O}

Available online 13 January 2011

\section{Keywords:}

Generalized Integral Transform Technique

Hybrid methods

Convection-diffusion

Non-linear problems

\begin{abstract}
A B S T R A C T
A unified approach for solving convection-diffusion problems using the Generalized Integral Transform Technique (GITT) was advanced and coined as the UNIT (UNified Integral Transforms) algorithm, as implied by the acronym. The unified manner through which problems are tackled in the UNIT framework allows users that are less familiar with the GITT to employ the technique for solving a variety of partial-differential problems. This paper consolidates this approach in solving general transient one-dimensional problems. Different integration alternatives for calculating coefficients arising from integral transformation are discussed. Besides presenting the proposed algorithm, aspects related to computational implementation are also explored. Finally, benchmark results of different types of problems are calculated with a UNIT-based implementation and compared with previously obtained results.
\end{abstract}

(c) 2011 Elsevier Ltd. All rights reserved.

\section{Introduction}

The Generalized Integral Transform Technique (GITT) [1-9] has been demonstrated to be a powerful tool for solving a variety of convection-diffusion problems. This technique is based on using orthogonal eigenfunctions expansions for expressing the unknown dependent variables; however, different from the Classical Integral Transform Technique [10], the transformation of the original problem needs not lead to a decoupled system, making the method applicable to a large number of problems.

The resulting transformed system is usually composed of a set of ODEs, which can be readily solved by well-established numerical routines that enable user-prescribed accuracy control. This, together with the analytical nature of this technique, allows for better global error control while compared to traditional domain discretization methods. The main drawback usually associated with the GITT is that a notable amount of analytical work can be required; nevertheless, this problem can be circumvented by the usage of symbolical computation $[7,9,11]$.

Some of the most recent applications of the Generalized Integral Transform Technique include, convective heat transfer in flows within wavy walls [12], hyperbolic heat conduction problems [13], conju-

\footnotetext{
is Communicated by W. J. Minkowycz.

* Corresponding author.

E-mail address: lasphaier@mec.uff.br (L.A. Sphaier).
}

gated conduction-convection problems [14], transient diffusion in heterogeneous media [15], heat and mass transfer in adsorption [16], atmospheric pollutant dispersion [17] and dispersion in rivers and channels [18], heat transfer in MHD [19], applications to irregular geometries [20], solution of the Navier-Stokes equations [21] and the boundary layer equations [22], stability analysis in natural convection [23], among others.

A characteristic aspect of all previous integral transform studies is that the solution strategy used in each work is tailored to the specific application. Although this can be very effective for individual analyses, when applying an existing solution strategy to a different problem, several adaptations could be necessary. This becomes particularly difficult for users not so well-versed with the Generalized Integral Transform Technique. Under this scenario, this contribution proposes a unified algorithm for solving virtually any convection-diffusion problem via Integral Transforms. This approach, named UNIT (UNified Integral Transforms), has the potential to enable a substantially greater number of users to apply the GITT for solving a variety of problems. Preliminary developments regarding the ideas behind this algorithm lead to two recent studies [24,25]. A first application of a preliminary UNIT algorithm was also used in the solution of the bioheat equation [26]. After these studies, the current work offers a consolidation of the unified integral transform approach, introducing the complete version of the one-dimensional UNIT algorithm in a formal mathematical fashion. In addition, simulation results of different test-problems are presented, providing a verification of the algorithm against previous literature results. 


\begin{tabular}{|ll|}
\hline Nomenclature \\
$C$ & concentration \\
$C_{j, n}$ & coefficients in semi-analytical integration \\
$\mathcal{D}$ & dispersion coefficient \\
$F$ & filter function \\
$f$ & initial condition function \\
$\bar{f}$ & transformed initial condition function \\
$G$ & prescribed source term \\
$g$ & modified source terms \\
$J$ & number of sub-regions in semi-analytical integration \\
$K$ & total number of governing equations in PDE system \\
$m$ & parameter in semi-analytical integration \\
$q$ & approximation order in semi-analytical integration \\
$T$ & dependent variables \\
& \\
& \\
Greek Symbols \\
$\mu$ & eigenvalues \\
$\Psi$ & eigenfunctions \\
$\Theta$ & filtered dependent variables \\
& \\
& \\
Subscripts & \\
$k$ & index for different equations and unknowns \\
()$^{*}$ & boundary condition parameter \\
& \\
&
\end{tabular}

\section{General one-dimensional transient problem}

In order to demonstrate the UNIT algorithm, a general onedimensional initial-value problem is considered. The problem comprises a system of $K$ coupled partial-differential equations and $K$ unknown potentials $T_{k}(x, t)$, written in the following form (for $\left.k=1, \ldots, K\right)$ :

$w_{k}(x) \frac{\partial T_{k}}{\partial t}=G_{k}(x, t, \boldsymbol{T}), \quad$ in $\quad x_{0} \leq x \leq x_{1}$, for $t>0$

$T_{k}(x, 0)=f_{k}(x), \quad$ in $\quad x_{0} \leq x \leq x_{1}$,

$\alpha_{k}(x) T_{k}(x, t)-(-1)^{n} \beta_{k}(x) k_{k}(x) \frac{\partial T_{k}}{\partial x}=\phi_{k}(x, t, \boldsymbol{T}), \quad$ at $\quad x=x_{n}$,

for $m=0,1$, where $\boldsymbol{T}$ is a vector containing the unknown potentials:

$\boldsymbol{T}=\left(T_{1}, \ldots, T_{k}, \ldots, T_{K}\right)$.

The $G_{k}$ functions carry all information regarding phenomena associated with combinations of spatial derivatives, and can depend on all unknown potentials in any arbitrary non-linear form. The purpose of writing the problem in the above form is that the user can provide any functional form for each of the $G_{k}$ functions. The functions $G_{k}$, as well as $f_{k}, \alpha_{k}, \beta_{k}, k_{k}$ and $\phi_{k}$ are the UNIT input parameters, and are known functions that can assume different forms according to the type of problem being solved.

The integral transform method involves the choice of eigenvalue problems that offer the basis for the eigenfunction expansions to be proposed. Therefore, an alternative form of the equation source terms can be written by explicitly showing the chosen diffusion and linear dissipation operators that are present in the auxiliary eigenvalue problem. Consequently, the modified source terms are defined for the inclusion of the chosen eigenvalue problem operators:

$g_{k}(x, t, \boldsymbol{T})=G_{k}(x, t, \boldsymbol{T})-\frac{\partial}{\partial x}\left(k_{k} \frac{\partial T_{k}}{\partial x}\right)+d_{k}(x) T_{k}$

With the previous definition, the governing PDEs in the initialvalue problem are rewritten as:

$w_{k}(x) \frac{\partial T_{k}}{\partial t}=\frac{\partial}{\partial x}\left(k_{k} \frac{\partial T_{k}}{\partial x}\right)-d_{k}(x) T_{k}+g_{k}(x, t, \boldsymbol{T})$.

Then, in order to remove unwanted convergence retardation effects associated with non-homogeneous terms, a set of filters can be introduced, based on the following solution representation:

$T_{k}(x, t)=\Theta_{k}(x, t)+F_{k}(x, t)$.

With the proposed general filtering, a filtered partial-differential system is obtained:

$w_{k}(x) \frac{\partial \Theta_{k}}{\partial t}=\frac{\partial}{\partial x}\left(k_{k} \frac{\partial \Theta_{k}}{\partial x}\right)-d_{k} \Theta_{k}+g_{k}^{*}(x, t, \boldsymbol{T}), \quad$ in $\quad x_{0} \leq x \leq x_{1}$,

$\alpha_{k}(x) \Theta_{k}(x, t)-(-1)^{n} \beta_{k}(x) k_{k}(x) \frac{\partial \Theta_{k}}{\partial x}=\phi_{k}^{*}(x, t, \boldsymbol{T}), \quad$ at $\quad x=x_{n}$,

$\Theta_{k}(x, 0)=f_{k}^{*}(x), \quad$ in $\quad x_{0} \leq x \leq x_{1}$,

for $n=0,1$, where the modified terms are given by:

$g_{k}^{*}(x, t, \boldsymbol{T})=g_{k}(x, t, \boldsymbol{T})+\frac{\partial}{\partial x}\left(k_{k} \frac{\partial F_{k}}{\partial x}\right)-d_{k}(x) F_{k}-w_{k}(x) \frac{\partial F_{k}}{\partial t}$,

$\phi_{k}^{*}(x, t, \boldsymbol{T})=\phi_{k}(x, t, \boldsymbol{T})-\alpha_{k}(x) F_{k}(x, t)-(-1)^{n} \beta_{k}(x) k_{k}(x) \frac{\partial F_{k}}{\partial x}$,

$f_{k}^{*}(x)=f_{k}(x)-F_{k}(x, 0)$.

Using Eqs. (3) and (7), one arrives at the following expression for the source terms $g_{k}^{*}$ as functions of the user-prescribed functional forms $G_{k}$ :

$g_{k}^{*}(x, t, \Theta)=G_{k}(x, t, \Theta+\boldsymbol{F})-\frac{\partial}{\partial x}\left(k_{k} \frac{\partial \Theta_{k}}{\partial x}\right)+d_{k}(x) \Theta_{k}-w_{k}(x) \frac{\partial F_{k}}{\partial t},(10$

where $\boldsymbol{\theta}$ is the vector with the filtered potentials and $\boldsymbol{F}$ is a vector with the filtering functions:

$\Theta=\left(\Theta_{1}, \ldots, \Theta_{k}, \ldots, \Theta_{K}\right)$

$\boldsymbol{F}=\left(F_{1}, \ldots, F_{k}, \ldots, F_{K}\right)$

\section{Eigenvalue problem and transformation pair}

The transformation of system (6a)-(6c) is accomplished using a general Sturm-Liouville eigenvalue problem for obtaining bases of orthogonal eigenfunctions:

$\frac{\mathrm{d}}{\mathrm{dx}}\left(k_{k}(x) \frac{\mathrm{d} \Psi_{k}}{\mathrm{~d} x}\right)+\left(\mu_{k}^{2} w_{k}(x)-d_{k}(x)\right) \Psi_{k}=0, \quad$ in $\quad x_{0} \leq x \leq x_{1}$,

$\alpha_{k}(x) \Psi_{k}(x)-(-1)^{n} \beta_{k}(x) k_{k}(x) \frac{\mathrm{d} \Psi_{k}}{\mathrm{~d} x}=0, \quad$ at $\quad x=x_{n}$, 
for $n=0,1$, in which the eigenfunctions are normalized through the following integrals:

$\int_{x_{0}}^{x_{1}} w_{k}(x) \tilde{\Psi}_{k, i}(x) \tilde{\Psi}_{k, j}(x) \mathrm{d} x=\delta_{i, j}, \quad$ for $\quad k=1, \ldots K$,

where $i$ and $j$ are integers, $\delta_{i, j}$ is the Kronecker delta function, and the normalized eigenfunctions are written as:

$\tilde{\Psi}_{k, i}(x)=\frac{\Psi_{k, i}(x)}{N_{k, i}^{1 / 2}}$,

and the normalization integral is given by:

$N_{k, i}=\int_{x_{0}}^{x_{1}} w_{k}(x) \Psi_{k, i}^{2}(x) \mathrm{d} x$.

Based on the orthonormality relations (14c), an integral transform pair is defined for each of the dependent variables:

$\bar{\Theta}_{k, i}(t)=\int_{x_{0}}^{x_{1}} w_{k}(x) \Theta_{k}(x, t) \tilde{\Psi}_{k, i}(x) \mathrm{d} x$,

$\Theta_{k}(x, t)=\sum_{i=1}^{\infty} \bar{\Theta}_{k, i}(t) \tilde{\Psi}_{k, i}(x)$

in which the eigenfunctions $\Psi_{k, i}(x)=\Psi_{k}\left(x, \mu_{k, i}\right)$ are obtained from solving Eqs. (13a)-(13b).

\section{Integral transformation}

Using the integral transform operators, defined in Eq. (15a), the filtered problem is transformed, leading to the following system of ODEs:

$\frac{\mathrm{d} \bar{\Theta}_{k, i}}{\mathrm{~d} t}=-\mu_{k, i}^{2} \bar{\Theta}_{k, i}(t)+\bar{b}_{k, i}^{*}(t, \Theta)+\bar{g}_{k, i}^{*}(t, \Theta)$,

$\bar{\Theta}_{k, i}(0)=\bar{f}_{k, i}^{*}$,

for $k=1, \ldots, K$. The quantities $\bar{b}_{k, i}^{*}, \bar{g}_{k, i}^{*}$ and $\bar{f}_{k, i}^{*}$ are given by:

$\bar{b}_{k, i}^{*}(t, \Theta)=-\left[\phi_{k}^{*}(x, t, \Theta) \frac{(-1)^{n} \tilde{\Psi}_{k, i}(x)+k_{k}(x) \tilde{\Psi}_{k, i}^{\prime}(x)}{\alpha_{k}(x)+\beta_{k}(x)}\right]_{x_{0}}^{x_{1}}$,

$\bar{g}_{k, i}^{*}(t, \Theta)=\int_{x_{0}}^{x_{1}} g_{k}^{*}(x, t, \Theta) \tilde{\Psi}_{k, i}(x) \mathrm{d} x$,

$\bar{f}_{k, i}^{*}=\int_{x_{0}}^{x_{1}} w_{k}(x) f_{k}^{*}(x) \tilde{\Psi}_{i, k}(x) \mathrm{d} x$

where the functions $\bar{g}_{k, i}^{*}$, are calculated from the original $G_{k}$ functions through the relation:

$\bar{g}_{k, i}^{*}(t, \Theta)=\int_{x_{0}}^{x_{1}}\left(G_{k}(x, t, \Theta+\boldsymbol{F})+w_{k}(x)\left(\sum_{j=1}^{\infty} \mu_{k, j}^{2} \bar{\Theta}_{k, j} \tilde{\Psi}_{k, j}(x)-\frac{\partial F_{k}}{\partial t}\right)\right) \tilde{\Psi}_{k, i}(x) \mathrm{d} x$,

in which $\boldsymbol{F}$ is given by Eq. (12) and the components of $\boldsymbol{\Theta}$ depend on the transformed potentials through the inversion relations (15b), such that:

$\Theta=\left(\sum_{i=1}^{\infty} \bar{\Theta}_{1, i}(t) \tilde{\Psi}_{1, i}(x), \ldots, \sum_{i=1}^{\infty} \bar{\Theta}_{k, i}(t) \tilde{\Psi}_{k, i}(x), \ldots, \sum_{i=1}^{\infty} \bar{\Theta}_{K, i}(t) \tilde{\Psi}_{K, i}(x)\right)$.
If the functional dependence of the input source term $G_{k}$ requires the evaluation of spatial derivatives, this is accomplished through the following relation:

$\frac{\partial^{n} \Theta_{k}}{\partial x^{n}}=\sum_{i=1}^{\infty} \bar{\Theta}_{k, i}(t) \frac{d^{n} \tilde{\Psi}_{k, i}}{\partial x^{n}}$.

In order to solve system (16a)-(16b), the summations are truncated to a finite number of terms $N$ (termed the truncation order), and numerically solved by an ODE solver with user-prescribed precision control. For the results calculated in this work the Mathematica function NDSolve was employed for this purpose. Once system (16a)-(16b) is solved, the original potentials can be recovered using the filter functions and inversion formula, leading to the relation:

$T_{k}(x, t)=F_{k}(x, t)+\sum_{j=1}^{\infty} \bar{\Theta}_{k, j}(t) \tilde{\Psi}_{k, j}(x)$

\section{Semi-analytical integration}

The calculation of the functions $\bar{g}_{k, i}^{*}$ and $\bar{f}_{k, i}^{*}$ require the computation of integrals involving the eigenfunctions. On a few occasions, some of these could be evaluated analytically; however, often this is not the case and numerical integration is usually required. The use of a general-purpose numerical integration scheme can present some drawbacks, especially for larger eigenvalues due to the highly oscillatory nature of the eigenfunctions. Therefore, in order to offer an alternative, a semi-analytical integration approximation scheme is proposed. Noting that the evaluation of $\bar{g}_{k, i}^{*}$ and $\bar{f}_{k, i}^{*}$ is accomplished by calculating integrals in the form

$\int_{x_{0}}^{x_{1}} h(x, t) \Psi_{i} \mathrm{~d} x$

where $h$ can be any continuous function, the following approximation is proposed:

$\int_{x_{0}}^{x_{1}} h(x, t) \Psi_{i} \mathrm{~d} x \approx \sum_{j=1}^{J} \int_{\hat{x}_{j-1}}^{\hat{x}_{j}} \hat{h}_{j}(x, t) \Psi_{i} \mathrm{~d} x$

where $J$ is the number of sub-regions and $x_{0}$ and $x_{1}$ are related through $\hat{x}_{j}$ by:

$x_{0}=\hat{x}_{0}, \quad x_{1}=\hat{x}_{J}$

In the above relations, each of the $\hat{h}_{j}$ functions is a polynomial approximation of $h$ within an interval $\hat{x}_{j} \leq x \leq \hat{x}_{j+1}$, that is:

$\hat{h}_{j}(x, t)=\sum_{m=0}^{q} C_{j, m}(t) x^{m}$,

where the $C_{j, m}(t)$ coefficients are calculated according to the type of approximation used, and $q$ is the order of the approximation. Naturally, this integration alternative considers that the integrals of $x^{m} \tilde{\Psi}(x)$ can be analytically evaluated. This semi-analytical integration scheme is thus aimed at providing an analytical evaluation of the oscillatory behavior of the eigenfunctions within each sub-interval, thereby avoiding excessive refinement required by the automatic numerical integration routines.

\section{Results and discussion}

After introducing the UNIT algorithm, the associated computational code implemented using mixed symbolical-numerical computation provided by the Mathematica system [11] is now demonstrated. Results 
for selected test-cases were obtained and compared with previous data from the literature.

\subsection{Non-linear Burgers equation}

The first example involves the solution of the non-linear Burgers equation with Dirichlet boundary conditions, as given by [4]:

$\frac{\partial T}{\partial t}+\left(u_{0}+b T\right) \frac{\partial T}{\partial x}=v \frac{\partial^{2} T}{\partial x^{2}}, \quad$ in $\quad 0 \leq x \leq 1$,

$T(0, t)=1 \quad$ and $\quad T(1, t)=0, \quad$ for $\quad t>0$,

$T(x, 0)=1, \quad$ for $0 \leq x \leq 1$

This problem corresponds to defining the UNIT solution parameters as:

$K=1, \quad x_{0}=0, \quad x_{1}=1$,

$G_{k}=v \frac{\partial^{2} T_{k}}{\partial x^{2}}-\left(u_{0}+b T_{k}\right) \frac{\partial T_{k}}{\partial x}$

$\alpha_{k}(0)=1, \quad \beta_{k}(0)=0, \quad \phi(0, t, T)=1$,

$\alpha_{k}(1)=1, \quad \beta_{k}(1)=0, \quad \phi_{k}(1, t, T)=0$,

$f_{k}(x)=1, \quad k_{k}(x)=1, \quad w_{k}(x)=1, \quad d_{k}(x)=0$.

A user-defined filter is used for this problem, being the solution of the steady-state linear version of the problem:

$F_{k}(x, t)=\frac{\mathrm{e}-\mathrm{e}^{x}}{\mathrm{e}-1}$

This problem is solved for typical values of the governing parameters $\left(u_{0}=1.0 ; b=0.5\right.$ and $\left.5.0 ; \nu=1.0\right)$, at $t=0.1$ and $t=0.5$, where the increase in the parameter $b$ is offered to enhance the non-linear effect in the formulation. Tables 1-4 illustrate the behavior of the UNIT solution, for $N=30$ terms in the eigenfunction expansion, and a gradually increasing number of sub-regions for the semi-analytical integration approximation $(J=60,120$, and 240). Also, three levels of function approximation for the calculation of integrals were considered: 0th, 1st and 2nd order polynomial forms. A comparison of the results for different values of $J$ and different approximation levels are employed for demonstrating the adequacy of the unified algorithm introduced in the present work. The results calculated with no integration approximation (fully analytical) are displayed for evaluating the precision of the semi-analytical approxima-

Table 1

Solution of non-linear Burgers equation with analytical and semi-analytical coefficient integration for $b=0.5, u_{0}=1, t=0.1$ and $N=30$.

\begin{tabular}{llllllll}
\hline$x$ & Order & $J=60$ & $J=120$ & $J=240$ & Analytical & $|\epsilon|$ & Ref. [4] \\
\hline 0.1 & 0th & 0.983518 & 0.984064 & 0.984340 & 0.984619 & $4.850 \times 10^{-6}$ & 0.98462 \\
& 1st & 0.984626 & 0.984620 & 0.984619 & & & \\
& 2nd & 0.984619 & 0.984619 & 0.984619 & & & \\
0.3 & 0th & 0.928904 & 0.930651 & 0.931536 & 0.932430 & $8.325 \times 10^{-7}$ & 0.93243 \\
& 1st & 0.932450 & 0.932435 & 0.932431 & & & \\
& 2nd & 0.932430 & 0.932430 & 0.932430 & & & \\
0.5 & 0th & 0.812347 & 0.815009 & 0.816358 & 0.817720 & $3.775 \times 10^{-7}$ & 0.81772 \\
& 1st & 0.817744 & 0.817726 & 0.817721 & & & \\
& 2nd & 0.817720 & 0.817720 & 0.817720 & & & \\
0.7 & 0th & 0.588812 & 0.591401 & 0.592712 & 0.594034 & $3.980 \times 10^{-6}$ & 0.59403 \\
& 1st & 0.594050 & 0.594038 & 0.594035 & & & \\
& 2nd & 0.594035 & 0.594034 & 0.594034 & & & \\
0.9 & 0th & 0.228736 & 0.229856 & 0.230421 & 0.230991 & $3.561 \times 10^{-5}$ & 0.23099 \\
& 1st & 0.230993 & 0.230991 & 0.230991 & & & \\
& 2nd & 0.230991 & 0.230991 & 0.230991 & & &
\end{tabular}

Table 2

Solution of non-linear Burgers equation with analytical and semi-analytical coefficient integration for $b=0.5, u_{0}=1, t=0.5$ and $N=30$.

\begin{tabular}{llllllll}
\hline$x$ & Order & $J=60$ & $J=120$ & $J=240$ & Analytical & $|\epsilon|$ & Ref. [4] \\
\hline 0.1 & 0th & 0.950191 & 0.950257 & 0.950291 & 0.950325 & $7.141 \times 10^{-7}$ & 0.95032 \\
& 1st & 0.950324 & 0.950324 & 0.950325 & & & \\
& 2nd & 0.950325 & 0.950325 & 0.950325 & & & \\
0.3 & 0th & 0.825596 & 0.825764 & 0.825849 & 0.825935 & $9.457 \times 10^{-7}$ & 0.82593 \\
& 1st & 0.825933 & 0.825934 & 0.825935 & & & \\
& 2nd & 0.825935 & 0.825935 & 0.825935 & & & \\
0.5 & 0th & 0.659742 & 0.659939 & 0.660038 & 0.660137 & $2.464 \times 10^{-7}$ & 0.66014 \\
& 1st & 0.660134 & 0.660137 & 0.660137 & & & \\
& 2nd & 0.660137 & 0.660137 & 0.660137 & & & \\
0.7 & 0th & 0.442458 & 0.442593 & 0.442660 & 0.442728 & $1.886 \times 10^{-6}$ & 0.44273 \\
& 1st & 0.442724 & 0.442727 & 0.442728 & & & \\
& 2nd & 0.442728 & 0.442728 & 0.442728 & & & \\
0.9 & 0th & 0.164231 & 0.164260 & 0.164274 & 0.164287 & $6.696 \times 10^{-6}$ & 0.16429 \\
& 1st & 0.164285 & 0.164287 & 0.164287 & & & \\
& 2nd & 0.164287 & 0.164287 & 0.164287 & & & \\
\hline
\end{tabular}

tions, and the results already reported in [4], corresponding to the GITT solution also with $N=30$ (with fully analytical integration), are included for verification purposes. A column with error estimates provided by the UNIT code is also presented, retaining the last three terms in the eigenfunction expansions. As can be seen, the UNIT results with analytical integration reconfirm the previously reported data.

When comparing the results from the approximate integration scheme with those obtained with exact analytical formulas, one notices that, for zeroth-order approximations, significant discrepancies can occur. Differences are generally seen in the second or third significant figure for $J=60$ divisions, and as the number of sub-regions is increased, a slow improvement is seen. In spite of the poor precision seen in zeroth-order approximations, when higher orders are used, a significant enhancement is achieved. First-order approximations, in general, yield four accurate digits for $J=60$, and at least five digits for $J=240$ divisions. Furthermore, when second-order approximations are used, six accurate digits can be obtained with only $J=60$ divisions.

\subsection{Non-linear diffusion}

The next test-problem is that of non-linear diffusion frequently encountered in heat conduction with temperature-dependent thermal conductivity [3]. A problem formulation with Neumann and Dirichlet boundary conditions is selected:

$\frac{\partial T}{\partial t}=\frac{\partial}{\partial x}\left(\left(k_{0}+b T\right) \frac{\partial T}{\partial x}\right), \quad$ in $\quad 0 \leq x \leq 1$,

Table 3

Solution of non-linear Burgers equation with analytical and semi-analytical coefficient integration for $b=5, u_{0}=1, t=0.1$ and $N=30$.

\begin{tabular}{llllllll}
\hline$x$ & Order & $J=60$ & $J=120$ & $J=240$ & Analytical & $|\epsilon|$ & Ref. [4] \\
\hline 0.1 & 0th & 0.998064 & 0.998288 & 0.998398 & 0.998506 & $8.520 \times 10^{-6}$ & 0.99851 \\
& 1st & 0.998511 & 0.998507 & 0.998506 & & & \\
& 2nd & 0.998506 & 0.998506 & 0.998506 & & & \\
0.3 & 0th & 0.986524 & 0.987751 & 0.988362 & 0.988972 & $3.935 \times 10^{-6}$ & 0.98897 \\
& 1st & 0.989000 & 0.988979 & 0.988974 & & & \\
& 2nd & 0.988972 & 0.988972 & 0.988972 & & & \\
0.5 & 0th & 0.940970 & 0.944309 & 0.946001 & 0.947708 & $1.191 \times 10^{-6}$ & 0.94771 \\
& 1st & 0.947760 & 0.947721 & 0.947711 & & & \\
& 2nd & 0.947709 & 0.947708 & 0.947708 & & & \\
0.7 & 0th & 0.782269 & 0.787697 & 0.790467 & 0.793276 & $9.435 \times 10^{-6}$ & 0.79328 \\
& 1st & 0.793277 & 0.793276 & 0.793276 & & & \\
& 2nd & 0.793277 & 0.793276 & 0.793276 & & & \\
0.9 & 0th & 0.349018 & 0.351644 & 0.352955 & 0.354263 & $5.931 \times 10^{-5}$ & 0.32646 \\
& 1st & 0.354163 & 0.354238 & 0.354256 & & & \\
& 2nd & 0.354263 & 0.354263 & 0.354263 & & &
\end{tabular}


Table 4

Solution of non-linear Burgers equation with analytical and semi-analytical coefficient integration for $b=5, u_{0}=1, t=0.5$ and $N=30$.

\begin{tabular}{llllllll}
\hline$x$ & Order & $J=60$ & $J=120$ & $J=240$ & Analytical & $|\epsilon|$ & Ref. [4] \\
\hline 0.1 & 0th & 0.996055 & 0.996313 & 0.996440 & 0.996567 & $7.215 \times 10^{-6}$ & 0.99657 \\
& 1st & 0.996572 & 0.996568 & 0.996567 & & & \\
& 2nd & 0.996567 & 0.996567 & 0.996567 & & & \\
0.3 & 0th & 0.976517 & 0.977762 & 0.978387 & 0.979013 & $3.459 \times 10^{-6}$ & 0.97901 \\
& 1st & 0.979036 & 0.979019 & 0.979015 & & & \\
& 2nd & 0.979014 & 0.979013 & 0.979013 & & & \\
0.5 & 0th & 0.916242 & 0.919223 & 0.920737 & 0.922267 & $1.061 \times 10^{-6}$ & 0.92227 \\
& 1st & 0.922299 & 0.922275 & 0.922269 & & & \\
& 2nd & 0.922267 & 0.922267 & 0.922267 & & & \\
0.7 & 0th & 0.742512 & 0.746756 & 0.748915 & 0.751099 & $8.59 \times 10^{-6}$ & 0.075110 \\
& 1st & 0.751070 & 0.751092 & 0.751097 & & & \\
& 2nd & 0.751099 & 0.751099 & 0.751099 & & & \\
0.9 & 0th & 0.323039 & 0.324768 & 0.325620 & 0.326462 & $5.517 \times 10^{-5}$ & 0.32646 \\
& 1st & 0.326367 & 0.326439 & 0.326456 & & & \\
& 2nd & 0.326462 & 0.326462 & 0.326462 & & & \\
\hline
\end{tabular}

$\left.\frac{\partial T}{\partial x}\right|_{x=0}=0$ and $\quad T(1, t)=0, \quad$ for $t>0$,

$T(x, 0)=1, \quad$ in $0 \leq x \leq 1$.

This corresponds to defining the UNIT solution parameters as:

$K=1, \quad x_{0}=0, \quad x_{1}=1$,

$G_{k}=\frac{\partial}{\partial x}\left(\left(k_{0}+b T_{k}\right) \frac{\partial T_{k}}{\partial x}\right)$

$\alpha_{k}(0)=0, \quad \beta_{k}(0)=1, \quad \phi_{k}(0, t, T)=0$,

$\alpha_{k}(1)=1, \quad \beta_{k}(1)=0, \quad \phi_{k}(1, t, T)=0$,

$f_{k}(x)=1 \quad k_{k}(x)=1, \quad w_{k}(x)=1, \quad d_{k}(x)=0$.

This problem is solved using the same UNIT code implementation used for the previous problem, the only modification being the previously indicated solution parameters. Tables 5 and 6 display the calculated solution for $b=5$ and $k_{0}=1$ in different instants $(t=0.1$ and $t=0.5$ ), using fully analytical integration as well as the semianalytical approximations. No filter was used for this case. Zeroth, first, and second-order approximations were used and different numbers of sub-regions $J$. As observed for the solution of Burgers equation, as naturally expected, the higher order approximations outperform the lower order ones, and, as the number of sub-regions is

Table 5

Solution of non-linear diffusion problem with analytical and semi-analytical coefficient integration for $b=5, k_{0}=1, t=0.1$ and $N=30$.

\begin{tabular}{llllllll}
\hline$x$ & Order & $J=60$ & $J=120$ & $J=240$ & Analytical & $|\epsilon|$ & Ref. [3] \\
\hline 0.1 & 0th & 0.578446 & 0.580004 & 0.580766 & 0.581349 & $3.29618 \times 10^{-5}$ & 0.58135 \\
& 1st & 0.581724 & 0.581445 & 0.581373 & & & \\
& 2nd & 0.581639 & 0.581355 & 0.581349 & & & \\
0.3 & 0th & 0.544484 & 0.546195 & 0.547050 & 0.547755 & $3.52761 \times 10^{-5}$ & 0.54775 \\
& 1st & 0.548132 & 0.547851 & 0.547779 & & & \\
& 2nd & 0.548036 & 0.547760 & 0.547755 & & & \\
0.5 & 0th & 0.473515 & 0.475509 & 0.476524 & 0.477428 & $6.62344 \times 10^{-5}$ & 0.47743 \\
& 1st & 0.477809 & 0.477525 & 0.477452 & & & \\
& 2nd & 0.477691 & 0.477433 & 0.477428 & & & \\
0.7 & 0th & 0.356648 & 0.359007 & 0.360233 & 0.361397 & $9.11878 \times 10^{-5}$ & 0.36140 \\
& 1st & 0.361784 & 0.361496 & 0.361422 & & & \\
& 2nd & 0.361628 & 0.361402 & 0.361397 & & & \\
0.9 & 0th & 0.165206 & 0.167667 & 0.168996 & 0.170351 & $1.40232 \times 10^{-3}$ & 0.17035 \\
& 1st & 0.170726 & 0.170447 & 0.170375 & & & \\
& 2nd & 0.170527 & 0.170355 & 0.170351 & & & \\
\hline
\end{tabular}

Table 6

Solution of non-linear diffusion problem with analytical and semi-analytical coefficient integration for $b=5, k_{0}=1, t=0.5$ and $N=30$.

\begin{tabular}{llllllll}
\hline$x$ & Order & $J=60$ & $J=120$ & $J=240$ & Analytical & $|\varepsilon|$ & Ref. [3] \\
\hline 0.1 & 0th & 0.121602 & 0.122682 & 0.123230 & 0.123761 & $2.92098 \times 10^{-6}$ & 0.12376 \\
& 1st & 0.123843 & 0.123782 & 0.123766 & & & \\
& 2nd & 0.123799 & 0.123762 & 0.123761 & & & \\
0.3 & 0th & 0.112224 & 0.113268 & 0.113797 & 0.114312 & $3.25075 \times 10^{-6}$ & 0.11431 \\
& 1st & 0.114389 & 0.114331 & 0.114316 & & & \\
& 2nd & 0.114348 & 0.114312 & 0.114312 & & & \\
0.5 & 0th & 0.093269 & 0.094215 & 0.094696 & 0.095165 & $6.05455 \times 10^{-6}$ & 0.09516 \\
& 1st & 0.095232 & 0.095182 & 0.095169 & & & \\
& 2nd & 0.095196 & 0.095166 & 0.095165 & & & \\
0.7 & 0th & 0.064272 & 0.065015 & 0.065394 & 0.065765 & $9.7277 \times 10^{-6}$ & 0.06576 \\
& 1st & 0.065814 & 0.065777 & 0.065768 & & & \\
& 2nd & 0.065787 & 0.065765 & 0.065765 & & & \\
0.9 & 0th & 0.024409 & 0.024748 & 0.024921 & 0.025093 & $1.50625 \times 10^{-4}$ & 0.02509 \\
& 1st & 0.025114 & 0.025098 & 0.025094 & & & \\
& 2nd & 0.025102 & 0.025093 & 0.025093 & & & \\
\hline
\end{tabular}

increased the results gradually converge to the analytical integration ones [3]. Nevertheless, when compared to the presented solution of Burgers equation, one sees that only the second-order approximation with 240 sub-regions matches the fully analytical integration with all six digits. For larger times $(t=0.5)$, a performance improvement is seen, with the second-order approximation matching all six digits obtained with the fully analytical integration for 120 sub-regions. For these larger instants, the first-order approximations present as much as 5 digits in agreement with the analytical integration results.

\subsection{Radionuclides decay chain}

The next example deals with the verification of object oriented numerical codes for systems of convection-diffusion equations in specific applications. In order to simulate the transport of radionuclides decay chains in soils, one must solve the coupled system of transport equations for each species in the chain for the hydrologic and geochemical conditions of the specific site being considered. Several powerful and well-tested simulation tools are publicly or commercially available for this purpose [27,28]. To solve the radionuclide transport problem, one-dimensional transient advection-diffusion equations are assumed for each of the $k=1, \ldots, K$ species in the radionuclide decay chain. The governing transport equations for the individual species are given by:

$R_{k} \frac{\partial C_{k}}{\partial t}+v \frac{\partial C_{k}}{\partial x}=\mathcal{D}_{k} \frac{\partial^{2} C_{k}}{\partial x^{2}}-\mu_{k} R_{k} C_{k}(x, t)+\mu_{k-1} R_{k-1} C_{k-1}(x, t), \quad$ in $\quad 0 \leq x \leq L$

where $C_{k}$ is concentration of each species, $t$ is the time, $x$ is distance, $L$ is the length of the transport domain, $v$ is the pore-water velocity, $\mathcal{D}_{k}$ are the dispersion coefficients, $R_{k}$ are the retardation factors, $\mu_{k}$ are the decay rates $\left(\mu_{0}=0\right.$ for the first radionuclide), and the subscript $k$ refers to the kth radionuclide in the decay chain. The associated initial and boundary conditions are:

$C_{k}(x, 0)=0, \quad$ in $\quad 0 \leq x \leq L$,

$-\gamma_{k} \mathcal{D}_{k} \frac{\partial C_{k}}{\partial x}+v C_{k}=v F_{k}(t)$, for $t>0$

$-\left.\mathcal{D}_{k} \frac{\partial C_{k}}{\partial x}\right|_{x=L}=0$, for $t>0$,

where $F_{k}(t)$ is the concentration of the kth radionuclide at the inlet. The boundary conditions at $x=0$ can be of third $\left(\gamma_{k} \neq 0\right)$ or first type 
$\left(\gamma_{k}=0\right)$, while at the exit boundary conditions are in general of second type.

The user-defined data for this problem are given by:

$G_{k}=\left(\mathcal{D}_{k} \frac{\partial^{2} C_{k}}{\partial x^{2}}-v \frac{\partial C_{k}}{\partial x}-\mu_{k} R_{k} C_{k}(x, t)+\mu_{k-1} R_{k-1} C_{k-1}(x, t)\right) / R_{k}$

$\alpha_{k}(0)=v, \quad \beta_{k}(0)=0, \quad \phi_{k}(0, t, T)=v F_{k}(t)$,

$\alpha_{k}(0)=0, \quad \beta_{k}(0)=1, \quad \phi_{k}(0, t, T)=0$,

$f_{k}(x)=0, \quad k_{k}(x)=1, \quad w_{k}(x)=1, \quad d_{k}(x)=0,$.

The UNIT algorithm was tested against two previously developed codes for radionuclides decay chains: the CHAIN code of [27] and the LBL (Lawrence Berkeley Laboratory) computer code of [28]. Solutions employed in these codes were derived using the Laplace transform technique as applied to semi-infinite media. The LBL code deals with purely advective transport (no dispersion) of radionuclides in saturated semi-infinite media to allow for explicit analytical inversion of the Laplace transforms. We consider here the three element LBL chain example formed by $\mathrm{U}^{234}, \mathrm{Th}^{230}$, and $\mathrm{Ra}^{226}$. The following parameter values were used: $\mathcal{D}=50 \mathrm{~m}^{2} /$ year, $v=1 \mathrm{~m} /$ year, $R_{1}=120, R_{2}=1500$, $R_{3}=300, \mu_{1}=2.806 \times 10^{-6}$ year $^{-1}, \mu_{2}=8.664 \times 10^{-6}$ year $^{-1}$, and $\mu_{3}=4.332 \times 10^{-4}$ year $^{-1}$. The normalized concentrations at boundary $x=0$ are given by $F_{1}=1, F_{2}=1$, and $F_{3}=10$. Numerical results were obtained for the same time values (10 and 1000 years) as used by [28], while we used a domain length $L=120 \mathrm{~m}$ to avoid any effects of the exit boundary.

Table 7 illustrates the convergence behavior of the eigenfunction expansion for $\mathrm{U}^{234}$ at $t=1000$ years. The selected truncation orders were $N=25,50$ and 75, while different numbers of sub-domains in the first-order semi-analytical integration were critically analyzed $(J=75,150$, and 225). Convergence is overall very satisfactory, yielding around five converged significant digits in the results in comparison to the analytical integration GITT results reported in [29], with $N=150$ terms in the expansions. One may observe the relatively small gain in precision achieved in the last set of results while increasing the truncation order to $N=75$ and the semi-analytical subdomains number to $J=225$. The GITT is then seen to be precise in predicting concentrations that are orders-of-magnitude smaller than those at the origin.

Finally, in order to illustrate the effect of using different approximation orders in the semi-analytical integration scheme, simulation results are performed for zeroth, first and second orders, as well as for different number of sub-regions, as depicted in Table 8. These results were obtained for $t=1000$ years and $N=40$ terms. As can be seen, the convergence is generally better for positions near $x=0$; however, there seems to be no effect of the position on the solution convergence with the number of sub-regions $(J)$ nor with the

Table 7

Convergence of the UNIT solution for the $\mathrm{U}^{234}$ concentration distribution at $t=1000$ years for the LBL test case of [28] involving $\mathrm{U}^{234}, \mathrm{Th}^{230}$ and $\mathrm{Ra}^{226}$.

\begin{tabular}{|c|c|c|c|c|c|c|}
\hline \multirow[b]{3}{*}{$x$} & \multicolumn{5}{|l|}{$N$} & \multirow[b]{3}{*}{ Ref. [29] } \\
\hline & 25 & 25 & 50 & 50 & 75 & \\
\hline & $J=25$ & $J=150$ & $J=75$ & $J=150$ & $J=225$ & \\
\hline 1 & 0.98072 & 0.98075 & 0.98085 & 0.98096 & 0.98094 & 0.98096 \\
\hline 10 & 0.79723 & 0.79723 & 0.79730 & 0.79729 & 0.79730 & 0.79730 \\
\hline 20 & 0.58592 & 0.58581 & 0.58595 & 0.58584 & 0.58582 & 0.58581 \\
\hline 30 & 0.39388 & 0.39373 & 0.39390 & 0.39375 & 0.39372 & 0.39369 \\
\hline 40 & 0.24076 & 0.24062 & 0.24077 & 0.24063 & 0.24060 & 0.24058 \\
\hline 60 & 0.06640 & 0.06637 & 0.06640 & 0.06637 & 0.06637 & 0.06636 \\
\hline 80 & 0.01195 & 0.01198 & 0.01195 & 0.01197 & 0.01197 & 0.01198 \\
\hline 100 & 0.00139 & 0.00140 & 0.00138 & 0.00139 & 0.00139 & 0.00139 \\
\hline
\end{tabular}

\section{Table 8}

Concentration $C_{1}$ for radionuclide decay chain problem with analytical and semianalytical coefficient integration for $t=1000$ and $N=40$.

\begin{tabular}{lllllll}
\hline$x$ & Order & $J=80$ & $J=160$ & $J=320$ & Analytical & $|\epsilon|$ \\
\hline 12. & 0th & 0.757337 & 0.756017 & 0.755367 & 0.754733 & $2.91 \times 10^{-5}$ \\
& 1st & 0.754770 & 0.754742 & 0.754735 & & \\
& 2nd & 0.754735 & 0.754735 & 0.754733 & & \\
30. & 0th & 0.394113 & 0.393609 & 0.393580 & 0.393689 & $3.40 \times 10^{-5}$ \\
& 1st & 0.393869 & 0.393734 & 0.393700 & & \\
& 2nd & 0.393691 & 0.393689 & 0.393689 & & \\
60. & 0th & 0.060485 & 0.063388 & 0.064869 & 0.066359 & $5.97 \times 10^{-5}$ \\
& 1st & 0.066391 & 0.066367 & 0.066361 & & \\
& 2nd & 0.066360 & 0.066359 & 0.066359 & & \\
90. & 0th & 0.002662 & 0.003494 & 0.003903 & 0.004312 & $6.41 \times 10^{-5}$ \\
& 1st & 0.004291 & 0.004307 & 0.004311 & & \\
& 2nd & 0.004313 & 0.004312 & 0.004312 & & \\
120. & 0th & 0.000040 & 0.000107 & 0.000145 & 0.000190 & $3.47 \times 10^{-2}$ \\
& 1st & 0.000186 & 0.000189 & 0.000190 & & \\
& 2nd & 0.000190 & 0.000190 & 0.000190 & & \\
\hline
\end{tabular}

order of approximation. By further examining the data, one notices that only the 2 nd order approximation matches the solution with fully analytical integration; as a matter of fact, on some instances this occurs for as little as $J=80$ divisions, thereby showing the superiority of this approximation over the other orders.

\section{Conclusions}

This paper presented a unified algorithm for solving partialdifferential systems using the Generalized Integral Transform Technique (GITT). The UNified Integral Transforms (or simply UNIT) approach, as implied by the coined acronym, was thus developed for handling a wide class of partial-differential problems in a unified way. This was accomplished by first grouping all spatial operators into a single source term. The main advantage of such approach is that a great part of the integral transformation process is carried out in one single operation. Second, this integral transformation is handled through a semianalytical integration scheme, which preserves the analytical evaluation of the oscillatory eigenfunctions integrals, and provides a flexible and cost-effective alternative to automatic numerical integration routines. Finally, the mixed symbolic-numerical implementation takes the advantage of the analytical nature of the methodology.

\section{Acknowledgment}

The authors would like to acknowledge the financial support provided by CNPq.

\section{References}

[1] R.M. Cotta, M.N. Özişik, Laminar forced convection inside ducts with periodic variation of inlet temperature, International Journal of Heat and Mass Transfer 29 (10) (1986) 1495-1501.

[2] R.M. Cotta, Hybrid numerical/analytical approach to nonlinear diffusion problems, Numerical Heat Transfer, Part B: Fundamentals 17 (2) (1990) 217-226.

[3] R. Serfaty, R.M. Cotta, Integral transform solutions of diffusion problems with nonlinear equation coefficients, International Communications in Heat and Mass Transfer 17 (6) (1990) 851-864.

[4] R. Serfaty, R.M. Cotta, Hybrid analysis of transient nonlinear convection-diffusion problems, International Journal of Numerical Methods for Heat and Fluid Flow 2 (1992) 55-62.

[5] R.M. Cotta, Integral Transforms in Computational Heat and Fluid Flow, CRC Press, Boca Raton, FL, 1993.

[6] R.M. Cotta, Benchmark results in computational heat and fluid flow:-the integral transform method, International Journal of Heat and Mass Transfer 37 (1) (1994) 381-394.

[7] R.M. Cotta, M.D. Mikhailov, Heat Conduction - Lumped Analysis, Integral Transforms, Symbolic Computation, John Wiley \& Sons, England, 1997.

[8] R.M. Cotta (Ed.), The Integral Transform Method in Thermal and Fluids Science and Engineering, Begell House, Inc, New York, 1998. 
[9] R.M. Cotta, M.D. Mikhailov, Hybrid methods and symbolic computations, in: W. Minkowycz, E.M. Sparrow, J.Y. Murthy (Eds.), Handbook of Numerical Heat Transfer, chap. 16, 2nd edn, John Wiley \& Sons, 2006.

[10] M.D. Mikhailov, M.N. Özișik, Unified Analysis and Solutions of Heat and Mass Diffusion, John Wiley \& Sons, New York, 1984

[11] S. Wolfram, The Mathematica Book, 5th edn.Wolfram Media/Cambridge University Press, New York/Champaign, IL, 2003.

[12] F.V. Castellões, J.N.N. Quaresma, R.M. Cotta, Convective heat transfer enhancement in low Reynolds number flows with wavy walls, International Journal of Heat and Mass Transfer 53 (9-10) (2010) 2022-2034.

[13] E.R. Monteiro, E.N. Macedo, J.N.N. Quaresma, R.M. Cotta, Integral transform solution for hyperbolic heat conduction in a finite slab, International Communications in Heat and Mass Transfer 36 (4) (2009) 297-303.

[14] C.P. Naveira, M. Lachi, R.M. Cotta, J. Padet, Hybrid formulation and solution for transient conjugated conduction-external convection, International Journal of Heat and Mass Transfer 52 (1-2) (2009) 112-123.

[15] C.P. Naveira-Cotta, R.M. Cotta, H.R.B. Orlande, O. Fudym, Eigenfunction expansions for transient diffusion in heterogeneous media, International Journal of Heat and Mass Transfer 52 (2009) 5029-5039.

[16] S.C. Hirata, P. Couto, L.G. Lara, R.M. Cotta, Modeling and hybrid simulation of slow discharge process of adsorbed methane tanks, International Journal of Thermal Sciences 48 (6) (2009) 1176-1183.

[17] G.L. Almeida, L.C.G. Pimentel, R.M. Cotta, Integral transform solutions for atmospheric pollutant dispersion, Environmental Modeling and Assessment 13 (1) (2008) 53-65.

[18] F.P.J. de Barros, R.M. Cotta, Integral transforms for three-dimensional steady turbulent dispersion in rivers and channels, Applied Mathematical Modelling 31 (2007) 2719-2732.

[19] J.A. Lima, J.N.N. Quaresma, E.N. Macêdo, Integral transform analysis of MHD flow and heat transfer in parallel-plates channels, International Communications in Heat and Mass Transfer 34 (2007) 420-431.

[20] L.A. Sphaier, R.M. Cotta, Analytical and hybrid solutions of diffusion problems within arbitrarily shaped regions via integral transforms, Computational Mechanics 29 (3) (2002) 265-276.
[21] G.G.C. de Lima, C.A.C. Santos, A. Haag, R.M. Cotta, Integral transform solution of internal flow problems based on Navier-Stokes equations and primitive variables formulation, International Journal for Numerical Methods in Engineering 69 (3) (2007) 544-561.

[22] S.P.A. Paz, E.N. Macedo, J.N.N. Quaresma, R.M. Cotta, Eigenfunction expansion solution for boundary-layer equations in cylindrical coordinates: simultaneously developing flow in circular tubes, Numerical Heat Transfer, Part A: Applications 52 (12) (2007) 1123-1149.

[23] L.S. de, B. Alves, R.M. Cotta, J. Pontes, Stability analysis of natural convection in porous cavities through integral transforms, International Journal of Heat and Mass Transfer 45 (6) (2002) 1185-1195.

[24] L.A. Sphaier, R.M. Cotta, C.P. Naveira-Cotta, J.N.N. Quaresma, The UNIT (Unified Integral Transforms) symbolic-numerical computational platform for benchmarks in convection-diffusion problems, Proceedings of the 30th IberianLatin-American Congress on Computational Methods in Engineering (CILAMCE), Buzios, RJ, Brazil, 2009.

[25] R.M. Cotta, J.N.N. Quaresma, L.A. Sphaier, C.P. Naveira-Cotta, Unified integral transform approach in the hybrid solution of multidimensional nonlinear convection-diffusion problems, Proceedings of the 14th International Heat Transfer Conference (IHTC), Washington D.C., USA., Paper no. 22396, 2010.

[26] R.M. Cotta, B.P. Cotta, C.P. Naveira-Cotta, G. Cotta-Pereira, Hybrid integral transforms analysis of the bioheat equation with variable properties, International Journal of Thermal Sciences 49 (9) (2010) 1510-1516.

[27] M.T. van Genuchten, Convective-dispersive transport of solutes involved in sequential first-order decay reactions, Computers and Geosciences 11 (1985) 129-147.

[28] H.C. Lung, P.L. Chambré, T.H. Pigford, W.W.L. Lee, Transport of Radioactive Decay Chains in Finite and Semi-Infinite Porous Media, Report LBL23987, Lawrence Berkeley Laboratory, Earch Sciences Division, 1987.

[29] R.M. Cotta, C.P. Naveira, M.T. van Genuchten, M.J. Ungs, An integral transforms application to environmental impact assessment of uranium mining waste disposal, Proc. of the 19th Brazilian Congress of Mechanical Engineering (COBEM), ABCM, Brasília, DF, Brazil, 2007. 